Population Study

Poster

Abstract ID: 130

\title{
Balance disorders among walk-in patients in the International Islamic University Malaysia (IIUM) Ear, Nose And Throat (ENT) clinic: An exploratory study
}

\author{
W. A. Wan Aslynn | Nurul Syarida Bt. Mohd Sakeri | Siti Aishah Bt Arshad \\ Department of Audiology and Speech-Language Pathology, Kulliyyah of Allied Health Sciences, \\ International Islamic University Malaysia
}

Introduction: The epidemiological studies of balance disorder symptoms in Malaysia are still understudied, therefore this research sought to explore this issue among the walk-in patients at the International Islamic University Malaysia (IIUM) Ear, Nose and Throat (ENT) clinic in Kuantan, Pahang. Methods: The study aimed to 1) observe the association of common balance disorder symptoms with factors like gender, ethnicity and age groups; 2) reveal the patterns of cases with each of the balance disorder symptom according to factors in 1); and 3) determine the pattern of balance disorders in this clinic. This study took place between February and April of 2016. The medical records of these patients were examined after services by ENT doctors. To extract the data, these documents were reviewed using a medical record review checklist. Results: A total of 92 medical records were reviewed. They consisted of $45.6 \%$ male and $54.4 \%$ female; Malay (60.8\%), Chinese (27.2\%), Indian (10.9\%) and others (1.1\%); these patients were from 3 months to 69 years old. Only $13(14.1 \%)$ out of these cases presented with balance disorders. The findings indicated no association ( $p>0.05$ ) between each of the balance symptom and the demographic factors. Out of the 13 cases; the most frequent symptoms observed were headache $(76.9 \%)$, imbalance $(53.8 \%)$ and vertigo $(46.2 \%)$. The peripheral vestibular disorder was found as the most common diagnosis of balance disorder; benign paroxysmal positional vertigo (BPPV) (4) and Meniere's disease (1). Both of the disorders showed vertigo, vomiting, headache and blur vision. Conclusions: It is crucial to understand the symptoms of balance disorders, particularly for a Malaysian population as it can enhance the clinical services and its quality of treatment or rehabilitation.

KEYWORDS: symptoms of balance, vestibular disorder, BPPV, Meniere's disease 